\title{
Completion of mixing matrices for non-closed social networks
}

\author{
Shu-Fang Hsu Schmitz and Carlos Castillo-Chavez
}

\begin{abstract}
The contact structure of a population is one of the main factors in disease transmission. It has a definite impact on the incidence rates of sexually-transmitted diseases in heterogeneously mixing populations. A social structure is defined through the categorization of individuals into different classes by some specified criteria. Once the criteria has been specified one can express the social structure as a mixing matrix with subject classes on one axis and partner classes on the other. Our framework allows partnerships between members of the targeted population and individuals of other populations. If we aggregate the unknown types of individuals into a single class, then the mixing matrix has one row missing. Mark-recapture methodology is used to conditionally estimate the size of the sexually active population of individuals who are not members of the targeted population. The completion of the two sex mixing matrix is carried out with the help of the two-sex mixing axioms introduced by Busenberg and Castillo-Chavez (1989, 1991) and Castillo-Chavez and Busenberg (1991). However, this approach does not give a unique solution with data from a single survey. To illustrate our approach we provide a detailed example using a heterosexually active population of college students.
\end{abstract}

1991 Mathematics Subject Classification: 92D30.

\section{Introduction}

Heterogeneity in dating behavior in a population may be influenced by several factors including age, income, religion, social status (Sattenspiel and Castillo-Chavez, 1990), sexual activity (Hethcote and Yorke, 1984), etc. Different criteria not only result in different mixing structures but may also give rise to distinct disease dynamics. The effect of a social/mixing structure on the dynamics of sexuallytransmitted diseases (STD's) such as HIV/AIDS, has been studied by Blythe and Anderson (1988a, 1988b), Blythe and Castillo-Chavez (1989), Busenberg and Castillo-Chavez (1989, 1991), Castillo-Chavez et al. (1989), Dietz (1988), Dietz and Hadeler (1988), Fredrickson (1971), Gupta et al. (1989), Hadeler (1989), Hethcote and Van Ark (1987), Hethcote and Yorke (1984), Huang et al. (1992), Hyman and Stanley (1989), Jacquez (1988), Jacquez et al. (1989) and Nold (1980). However, the populations modeled in these studies not only lived in very simple social structures but also were not capable of interacting with others, that is, the populations were assumed to be "completely" closed. This assumption is not generally true. 
People travel continuosly within and between countries and these behaviors have obviously influenced the global pattern of spread of HIV/AIDS. At some temporal scales, the assumption of a closed interacting population may lead to misleading conclusions.

A survey of social and sexual patterns among college students (Crawford et al. 1990) reveals that about $50 \%$ of the heterosexual relationships among college students involve non-college-student sex partners which we refere to as the other or the unknown class (Castillo-Chavez et al., 1992). Obviously the study of the mixing/dating patterns within a college is not sufficient to understand STD epidemics in more representative populations. Here we use the data on this population with two purposes in mind: to show that it is possible to begin to quantify the mixing structure of a population, and to present an example of how a real population actually mixes. The lack of direct information on subjects in the other class makes it difficult to complete a social mixing matrix that takes into account this external population. The completion of a mixing matrix requires the estimation of many parameters including the size of the sexually active population of the other class as well as the estimation of the proportions of relationships that individuals in the other class have with individuals of all classes (the elements of the last row of the mixing matrix). The former task can be done by using mark-recapture methodology as shown by Rubin et al. (1992) while the latter can be reduced to the estimation of a single parameter through the application of the two-sex mixing formalism of Busenberg and Castillo-Chavez $(1989,1991)$ and Castillo-Chavez and Busenberg (1991). This paper illustrates the estimating procedure for these two tasks using dating data from a population of interacting college students.

'The organization of this paper is as follows. In Section 2, the background and source of the data are described and some relevant summary statistics are presented. Two separate incomplete mixing matrices for males and females are constructed using these data. In Section 3, mark-recapture methodology is used to conditionally estimate the active population size of the other class. In Section 4, the two-sex mixing framework is introduced and used to reduce the completion of the social structure to the estimation of a single parameter. In Section 5, we discuss the implications of our results and future directions.

\section{Data}

The data are extracted from a survey conducted among a population of college students (Crawford et al., 1990). Subjects (respondents) are categorized into four classes: 1 (freshman), 2 (sophomore), 3 (junior), and 4 (senior). Their partners are categorized into five classes, where the first four classes are the same as the ones for the subjects, and the 5th class is designated as the other class. This class includes partners who do not belong to the surveyed population. We use superscripts ${ }^{m}$ and ${ }^{f}$ to indicate male and female populations, and use subscripts $i$ and ${ }_{j}$ to indicate their class, respectively. Exact population sizes are denoted by 
$R_{i}^{m}$ and $R_{j}^{f}$. For the respondent population of students, these population sizes are available from the university's registrar office. The sample sizes denoted by $S_{i}^{m}$ and $S_{j}^{f}$ count the respondents; while the active sample sizes denoted by $A_{i}^{m}$ and $A_{j}^{f}$ count the respondents who were active in dating during the two-month period (our unit of time) prior to the survey. For each subject class, the total number of partners is denoted by $Y_{i}^{m}$ or $Y_{j}^{f}$, while its distribution among partner classes is denoted by $U_{i j}^{m}$ or $U_{j i}^{f}$. The within campus partnerships are denoted by $X_{i}^{m}$ and $X_{j}^{f}$ and are calculated by summing the partnerships with classes 1 through 4 . Division of $U_{i j}^{m}$ or $U_{j i}^{f}$ by the corresponding $Y_{i}^{m}$ or $Y_{j}^{f}$ gives an estimate of the mixing proportions, here denoted by $P_{i j}^{m}$ or $P_{j i}^{f}$. Estimates for the average numbers of partners for active subjects are denoted by $C_{i}^{m}$ and $C_{j}^{f}$. They are derived by dividing $Y_{i}^{m}$ and $Y_{j}^{f}$ by $A_{i}^{m}$ and $A_{j}^{f}$, respectively.

Table 1 summarizes the population sizes, sample sizes, active sample sizes, active proportions in the samples, and estimated active population sizes (denoted by $T_{i}^{m}$ and $T_{j}^{f}$ ) for both males and females in each of the four subject classes as well as the sum over the four respective classes. Each estimated active population size is calculated by multiplying the population size by the active proportion in the sample. Among those respondents, about $70 \%$ were active in dating. The overall active proportion for females $(73.2 \%)$ is a little higher than that for males $(69.6 \%)$. The partnership distribution, mixing proportions and average number of partners are presented in Table 2 and Table 3 . The row total of the mixing proportions may not be exactly equal to 1 because of rounding. For active males, the overall proportion of partuerships with class 5 is $23.0 \%$, while the overall average number of partners is 3.32. The corresponding values for active females are $28.3 \%$ and 2.66. It seems females take more dating partners from the other class and on the average have less partners than males from the respondent population. These two mixing matrices are also plotted in Figure 1 and Figure 2. One sees a like-withlike mixing pattern within the first four classes for both genders, namely, freshmen prefer freshmen, sophomores prefer sophomores, etc. Clearly, random mixing is not the case here. The next substantial mixing effect clearly comes from class 5 . One also notices that males mix more with females in the same or lower classes and females mix more with males in the same or higher classes, which is reasonable as in our data classes are positively correlated with age.

We observe that one row is missing (five elements, $P_{51}^{m}-P_{55}^{m}$, or $P_{51}^{f}-P_{55}^{f}$ ) in each of the above mixing matrices. Furthermore, estimates of the active population sizes $T_{5}^{m}$ and $T_{5}^{f}$ and average numbers of partners $C_{5}^{m}$ and $C_{5}^{f}$ in class 5 are also missing. The following sections will outline our approach for the estimation of these parameters. 
Table 1. Population sizes and sample sizes for males/females

\begin{tabular}{cccccc}
\hline $\begin{array}{c}\text { Class } \\
i / j\end{array}$ & $\begin{array}{c}\text { Popn. Size } \\
R\end{array}$ & $\begin{array}{c}\text { Sample Size } \\
S\end{array}$ & $\begin{array}{c}\text { Act. Sample Size } \\
A\end{array}$ & $\begin{array}{c}\text { Act. Prop. } \\
A \div S\end{array}$ & $\begin{array}{c}\text { Act. Popn. Size } \\
T\end{array}$ \\
\hline 1 & $1673 / 1278$ & $79 / 66$ & $56 / 44$ & $0.709 / 0.667$ & $1186 / 852$ \\
2 & $1589 / 1308$ & $60 / 57$ & $38 / 45$ & $0.633 / 0.789$ & $1006 / 1033$ \\
3 & $1591 / 1277$ & $38 / 51$ & $29 / 39$ & $0.763 / 0.765$ & $1214 / 977$ \\
4 & $1686 / 1348$ & $73 / 65$ & $51 / 47$ & $0.699 / 0.725$ & $1178 / 975$ \\
\hline Total & $6539 / 5211$ & $250 / 239$ & $174 / 175$ & $0.696 / 0.732$ & $4584 / 3837$ \\
\hline
\end{tabular}

Table 2. Dating partnerships distribution $U_{i j}^{m}$, mixing proportions $\left(P_{i j}^{m}\right)$ and average number of partners $C_{i}^{m}$ for male respondents

\begin{tabular}{crrrrrrrc}
\hline Class & & Female & Partner & Class & $j$ & & Total & Average \\
$i$ & 1 & 2 & 3 & 4 & $X_{i}^{m}$ & 5 & $Y_{i}^{m}$ & $C_{i}^{m}$ \\
\hline 1 & 123 & 26 & 15 & 4 & 168 & 50 & 218 & 3.89 \\
& $(0.564)$ & $(0.119)$ & $(0.069)$ & $(0.018)$ & & $(0.229)$ & & \\
2 & 23 & 53 & 20 & 7 & 103 & 25 & 128 & 3.37 \\
& $(0.180)$ & $(0.414)$ & $(0.156)$ & $(0.055)$ & & $(0.195)$ & & \\
3 & 11 & 19 & 27 & 15 & 72 & 19 & 91 & 3.14 \\
& $(0.121)$ & $(0.209)$ & $(0.297)$ & $(0.165)$ & & $(0.209)$ & & \\
4 & 11 & 11 & 27 & 53 & 102 & 39 & 141 & 2.76 \\
& $(0.078)$ & $(0.078)$ & $(0.191)$ & $(0.376)$ & & $(0.277)$ & & \\
\hline Total & 168 & 109 & 89 & 79 & 445 & 133 & 578 & 3.32 \\
& $(0.291)$ & $(0.189)$ & $(0.154)$ & $(0.137)$ & $X_{+}^{m}$ & $(0.230)$ & $Y_{+}^{m}$ & \\
\hline
\end{tabular}

Table 3. Dating partnerships distribution $U_{j i}^{f}$, mixing proportions $\left(P_{j i}^{f}\right)$ and average number of partners $C_{j}^{f}$ for female respondents

\begin{tabular}{crrrrrrrc}
\hline Class & & Male & Partner & Class & $i$ & & Total & Average \\
$j$ & 1 & 2 & 3 & 4 & $X_{j}^{f}$ & 5 & $Y_{j}^{f}$ & $C_{j}^{f}$ \\
\hline 1 & 58 & 17 & 6 & 9 & 90 & 24 & 114 & 2.59 \\
& $(0.509)$ & $(0.149)$ & $(0.053)$ & $(0.079)$ & & $(0.211)$ & & \\
2 & 9 & 45 & 27 & 15 & 96 & 33 & 129 & 2.87 \\
& $(0.070)$ & $(0.349)$ & $(0.209)$ & $(0.116)$ & & $(0.256)$ & & \\
3 & 2 & 10 & 41 & 22 & 75 & 22 & 97 & 2.49 \\
& $(0.021)$ & $(0.103)$ & $(0.423)$ & $(0.227)$ & & $(0.227)$ & & \\
4 & 2 & 6 & 20 & 45 & 73 & 53 & 126 & 2.66 \\
& $(0.016)$ & $(0.048)$ & $(0.159)$ & $(0.357)$ & & $(0.421)$ & & \\
\hline Total & 71 & 78 & 94 & 91 & 334 & 132 & 466 & 2.66 \\
& $(0.152)$ & $(0.167)$ & $(0.202)$ & $(0.195)$ & $X_{+}^{f}$ & $(0.283)$ & $Y_{+}^{f}$ & \\
\hline
\end{tabular}




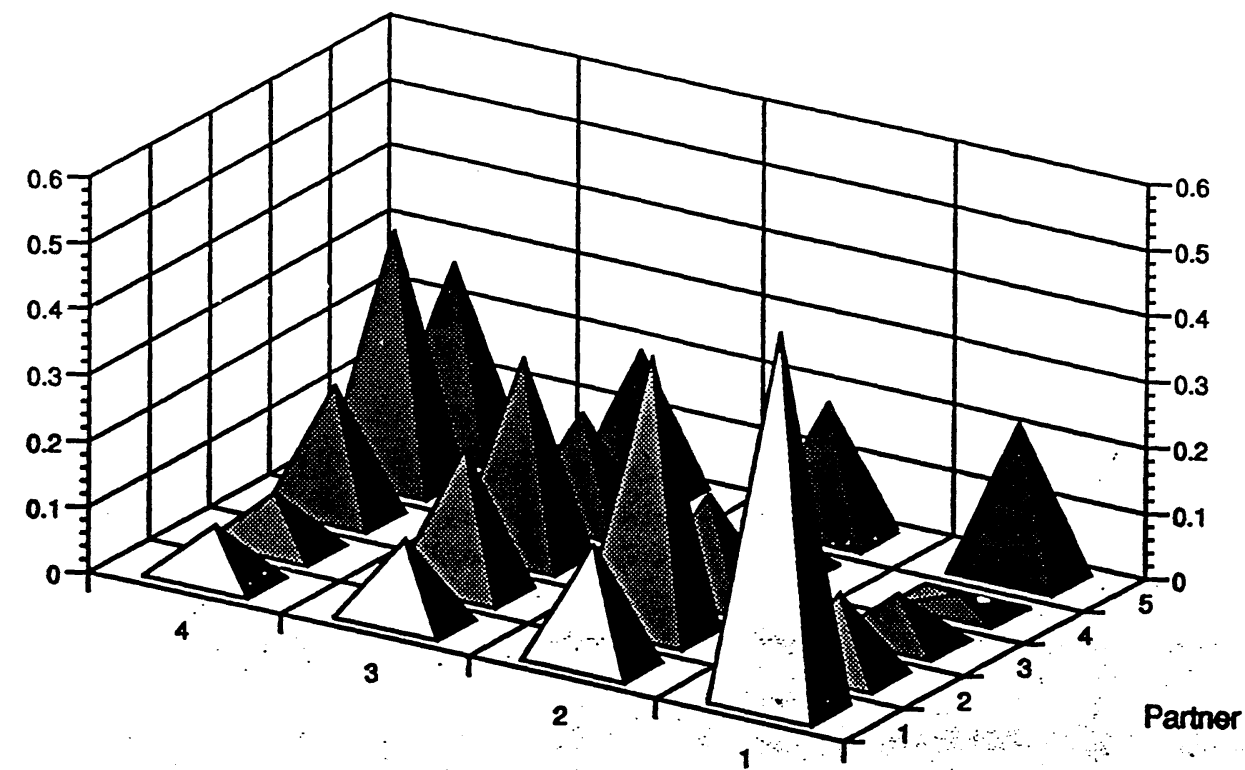

Respondent

Figure 1. Dating pattern of male respondents

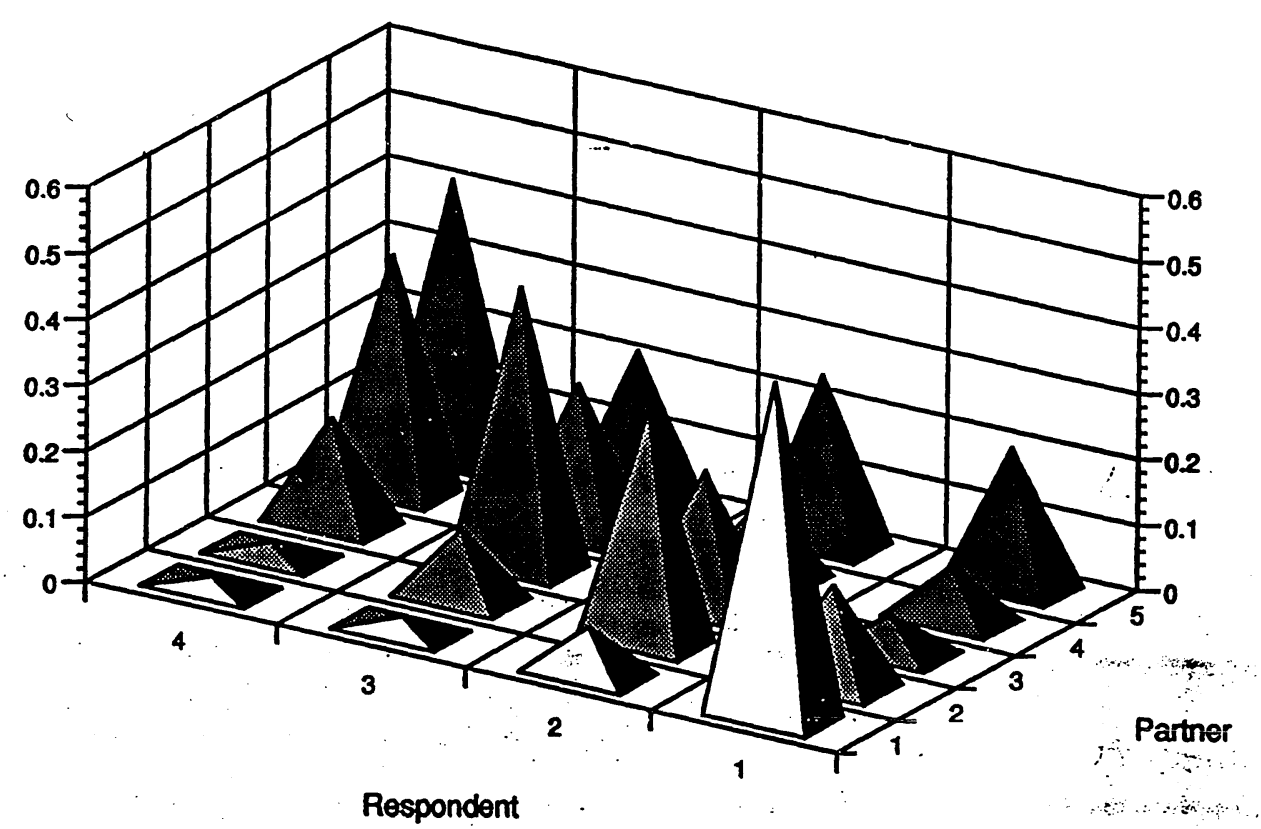

Figure 2. Dating pattern of female respondents 


\section{Mark-recapture methodology}

In wildlife study, mark-recapture or capture-recapture techniques are used extensively to estimate the size of an animal population (Seber, 1982). The simplest model is based on two samples. In the first sample animals from the population under consideration are captured, marked and then released. The second sample, after a certain time, re-captures some individuals from the same population. The number of marked individuals in the second sample are counted. Then a probability model such as the hypergeometric or the binomial distribution is used to estimate the population size. For our data and purposes, this technique has been adjusted. The total active population of undergraduates in class 1 through class 4 acts as the first sample. Individuals of this population are self-marked with the mark "student of the university". Instead of physical recapture, the second sample is collected by "sighting" from observers, the active respondents of the other gender. That is, female partners of male active respondents constitute the second sample for the female active population, while male partners of female active respondents constitute the second sample for the male active population. The resulting estimated population size from the model is in fact the estimated total active population size for all five classes. Due to recapture by "sighting", only those active indivduals who dated respondents are "sighted". Those who were not members of the surveyed population, were active in dating, but had no partners from the surveyed population are not estimable by this method. Hence, the definition of class 5 is restricted by the above "sighting" condition.

Since different oberservers might sight the same individual, that is, they may have shared the same partner, we assume that the second sampling is done with replacement. Therefore, the binomial model, not the most commonly used hypergeometic model, is preferred (Rubin et al., 1992). Bailey (1951) computes the estimator of population size for the binomial model and points out that it is much less biased than the Lincoln-Petersen's estimator for the hypergeometric model. $\mathrm{He}$ also provides the variance for his estimator. However, since our first sample size is also an estimate $\left(T_{+}^{m} \equiv T_{1}^{m}+T_{2}^{m}+T_{3}^{m}+T_{4}^{m}\right.$ for males, $T_{+}^{f} \equiv T_{1}^{f}+T_{2}^{f}+T_{3}^{f}+T_{4}^{f}$ for females), we have extra variability in our estimate of the total active population size for all five classes (denoted by $N^{m}$ and $N^{f}$ ). Rubin et al. (1992) modified the estimate for the variance of Bailey's estimator to take into account this variability.

As in $T_{+}^{m}$ and $T_{+}^{f}$, the subscript + represents the sum over the first four classes, i.e., the marked individuals. So $Y_{+}^{m}$ and $Y_{+}^{f}$ denote the total partnerships for all respondents, and $X_{+}^{m}$ and $X_{+}^{f}$ denote the total marked partners for all respondents. Using Bailey's estimator, one finds that the total active population sizes for the five classes are

$$
\begin{gathered}
N^{m}=\frac{T_{+}^{m} \times\left(Y_{+}^{f}+1\right)}{X_{+}^{f}+1}=6390 \quad(\text { male }) \\
N^{f}=\frac{T_{+}^{f} \times\left(Y_{+}^{m}+1\right)}{X_{+}^{m}+1}=4981 \quad(\text { female }) .
\end{gathered}
$$


Since the estimated total active population sizes for the marked individuals are known, the estimated active population size for the 5th class, or the unmarked individuals, can be obtained by subtraction. Hence,

$$
\begin{aligned}
T_{5}^{m} & =N^{m}-T_{+}^{m}=1806 \quad(\text { male }), \\
T_{5}^{f} & =N^{f}-T_{+}^{f}=1144 \quad(\text { female }) .
\end{aligned}
$$

With these estimated population sizes we can apply the two-sex mixing axioms to complete the mixing matrices. This is the topic of the next section.

\section{Completion of the mixing matrices}

Castillo-Chavez and Busenberg (1991) state that in a closed mixing population, the elements of the mixing matrices which describe the interaction between subpopulations satisfy the following two-sex mixing axioms at all time:

(A1) $\quad 0 \leq P_{i j}^{m} \leq 1, \quad 0 \leq P_{j i}^{f} \leq 1$.

(A2) $\quad \sum_{j} P_{i j}^{m}=1=\sum_{i} P_{j i}^{f}$.

(A3) $C_{i}^{m} T_{i}^{m} P_{i j}^{m}=C_{j}^{f} T_{j}^{f} P_{j i}^{f}$.

In our example, $i, j=1,2,3,4,5$, but $C_{5}^{m}, C_{5}^{f}, P_{51}^{m}-P_{55}^{m}, P_{51}^{f}-P_{55}^{f}$ are not known. To obtain point estimates for these parameters and mixing elements, we let $i=5$ and sum over $j$ on both sides of (A3) to get

$$
C_{5}^{m} T_{5}^{m}=K_{5}^{f}+P_{55}^{f} C_{5}^{f} T_{5}^{f}
$$

where $K_{5}^{f}=\sum_{j=1}^{4} P_{j 5}^{f} C_{j}^{f} T_{j}^{f}$ is known. Similarly, we let $j=5$ and sum over $i$ on both sides of (A3) to get

$$
C_{5}^{f} T_{5}^{f}=K_{5}^{m}+P_{55}^{m} C_{5}^{m} T_{5}^{m},
$$

where $K_{5}^{m}=\sum_{i=1}^{4} P_{i 5}^{m} C_{i}^{m} T_{i}^{m}$ is also known. Rearranging of equations (1) and (2) leads to lower bounds for $C_{5}^{m}$ and $C_{5}^{f}$. First we note that

$$
\begin{aligned}
P_{55}^{f} C_{5}^{f} T_{5}^{f} & =C_{5}^{m} T_{5}^{m}-K_{5}^{f} \geq 0, \\
P_{55}^{m} C_{5}^{m} T_{5}^{m} & =C_{5}^{f} T_{5}^{f}-K_{5}^{m} \geq 0 ;
\end{aligned}
$$

and hence

$$
\begin{array}{ll}
C_{5}^{m} \geq K_{5}^{f} / T_{5}^{m} \quad(=1.59 \quad \text { in our example }) \\
C_{5}^{f} \geq K_{5}^{m} / T_{5}^{f} \quad(=2.99 \quad \text { in our example }) .
\end{array}
$$

Since only active (e.g.,dating) individuals who had at least one partner are under consideration, we know that $C_{5}^{m}$ and $C_{5}^{f}$ are greater than or equal to 1 . Therefore,

$$
\begin{aligned}
C_{5}^{m} & \geq \max \left(K_{5}^{f} / T_{5}^{m}, 1\right), \\
C_{5}^{f} & \geq \max \left(K_{5}^{m} / T_{5}^{f}, 1\right) .
\end{aligned}
$$


Subtracting equation (2) from equation (1), we obtain the following linear relationship between $C_{5}^{m}$ and $C_{5}^{f}$ :

$$
C_{5}^{m} T_{5}^{m}-C_{5}^{f} T_{5}^{f}=K_{5}^{f}-K_{5}^{m} \equiv K,
$$

where $P_{55}^{f} C_{5}^{f} T_{5}^{f}-P_{55}^{m} C_{5}^{m} T_{5}^{m}$ vanishes by axiom (A3). Our data give the following explicit linear relationship:

$$
0.000874 C_{5}^{m}-0.000554 C_{5}^{f}=-0.000268
$$

and hence

$$
C_{5}^{f}=1.577617 C_{5}^{m}+0.483755>C_{5}^{m} .
$$

Consistency demands that the average number of partners of females in class 5 must be larger than that of males in class 5 . The situation is opposite to that of the first four classes.

Due to insufficient data, there is no unique solution. We need to guess appropriate values for $C_{5}^{m}$ or $C_{5}^{f}$ in the absence of independent estimators. Suppose $C_{5}^{m *}$ is the appropriate value for $C_{5}^{m}$, then $C_{5}^{f *}$ can be uniquely obtained by equation (5). Plugging these values into equations (1) and (2) specifies the values of $P_{55}^{f}$ and $P_{55}^{m}$ since

$$
P_{55}^{f *}=\left(C_{5}^{m *} T_{5}^{m}-K_{5}^{f}\right) / C_{5}^{f *} T_{5}^{f}
$$

and

$$
P_{55}^{m *}=\left(C_{5}^{f *} T_{5}^{f}-K_{5}^{m}\right) / C_{5}^{m *} T_{5}^{m} .
$$

Point estimates for $P_{51}^{m *}-P_{54}^{m *}$ are obtained by using axiom (A3):

$$
P_{5 j}^{m *}=P_{j 5}^{f} C_{j}^{f} T_{j}^{f} / C_{5}^{m *} T_{5}^{m} .
$$

Similarly, $P_{51}^{f *}-P_{54}^{f *}$ are given by

$$
P_{5 i}^{f *}=P_{i 5}^{m} C_{i}^{m} T_{i}^{m} / C_{5}^{f *} T_{5}^{f} .
$$

Table 4 presents the results obtained by using different $C_{5}^{m *}$ values. By construction, $P_{5 j}^{m *}$ and $P_{5 i}^{f *}$ satisfy axioms (A1), (A2) and (A3). We also observe that $C_{5}^{f *}$ gets larger as $C_{5}^{m *}$ gets larger, which is a direct consequence of (6); also $P_{55}^{m *}$ and $P_{55}^{f *}$ increase simultaneously forcing the remaining $P_{5 j}^{m *}$ and $P_{5 i}^{f *}$ to shrink by axiom (A2).

\section{Conclusions}

Our data indicate that about $20 \%$ of the heterosexually dating partnerships for undergraduate students are with individuals who do not belong to the surveyed population, the other class. The proportion may be too high to be ignored. However, here we have not yet resolved the problem completly. The estimated active 
Table 4. Estimated mixing proportions for males $P_{5 j}^{m *}$

(upper line) and females $P_{5 i}^{f *}$ (lower line) in class 5

\begin{tabular}{|c|c|c|c|c|c|c|}
\hline$C_{5}^{m *}$ & $C_{5}^{f *}$ & 1 & $\begin{array}{c}\text { Partner } \\
2\end{array}$ & $\begin{array}{c}\text { Class } \\
3\end{array}$ & $\begin{array}{c}j \text { or } i \\
4\end{array}$ & 5 \\
\hline 1.60 & 3.01 & $\begin{array}{l}0.161 \\
0.307\end{array}$ & $\begin{array}{l}0.262 \\
0.192\end{array}$ & $\begin{array}{l}0.191 \\
0.231\end{array}$ & $\begin{array}{l}0.377 \\
0.262\end{array}$ & $\begin{array}{l}0.009 \\
0.008\end{array}$ \\
\hline 2.50 & 4.43 & $\begin{array}{l}0.103 \\
0.209\end{array}$ & $\begin{array}{l}0.168 \\
0.131\end{array}$ & $\begin{array}{l}0.122 \\
0.157\end{array}$ & $\begin{array}{l}0.241 \\
0.178\end{array}$ & $\begin{array}{l}0.366 \\
0.326\end{array}$ \\
\hline 5.00 & 8.38 & $\begin{array}{l}0.051 \\
0.110\end{array}$ & $\begin{array}{l}0.084 \\
0.069\end{array}$ & $\begin{array}{l}0.061 \\
0.083\end{array}$ & $\begin{array}{l}0.121 \\
0.094\end{array}$ & $\begin{array}{l}0.683 \\
0.644\end{array}$ \\
\hline
\end{tabular}

population sizes for the other class from mark-recapture methodology are conditional on the fact that individuals in the class must have had at least one partner from the targeted population. This conditional estimation is certainly useful for some STD's when a core group of highly active individuals or a population of prostitutes is key to the transmission dynamics (Hethcote and Yorke, 1984). Individuals in the core group are considered as marked. Their partners are either marked or unmarked. Then the mark-recapture methodology can be used to estimate the total population size of individuals at risk of STD's.

Based on the two-sex mixing axioms, the estimation of missing elements of the mixing matrices is reduced to a single-parameter problem. This key parameter is the average number of partners for active males or females in the other class. Once this parameter is evaluated, then the mixing matrices can be completed.

To illustrate our approach, we used five classes for the mixing structure of the example. Clearly, the estimation procedures described here work for any finite number of classes. However, there are some practical considerations: too few classes may not give a clear picture of the mixing structure, while too many classes will add parameters making the estimation procedure more difficult. For example, some elements of the mixing matrices may be close to zero. Our experience suggests that the use of four to six classes per sexual orientation is optimal. Finally, we point out the fact that we have only obtained point estimates for the mixing matrix. A complete characterization of such complex structure can only be accomplished with the aid of extensive cross-sectional and longitudinal data. The data used here gives however a clear indication that observational studies may be able to give a good description of this complex process. That is, females prefer to mix with older males and more frequently with males of external populations which have to be estimated as they may play (as in the example provided above) a fundamental role in explaining the inadequacies of survey data. Also the estimate will help us to develop more accurate instruments for further study. 


\section{Acknowledgements}

This research has been partially supported by NSF grant DEB-9253570, and Hatch project grant NYC 151-409, USDA to CC-C. We thank professor Fred Brauer for his valuable comments.

\section{References}

[1] Bailey, N.T.J., On estimating the size of mobile populations from recapture data. Biometrika 38 (1951), 293-306.

[2] Blythe, S.P., R.M. Anderson, Distributed incubation and infectious periods in models of the transmission dynamics of the human immunodeficiency virus (HIV). IMA J. Math. Appl. Med. Biol. 5 (1988a), 1-19.

[3] Blythe, S.P., R.M. Anderson, Variable infectiousness in HIV transmission models. IMA J. Math. Appl. Med. Biol. 5 (1988b), 181-200.

[4] Blythe, S.P., C. Castillo-Chavez, Like-with-like preference and sexual mixing models. Math. Biosci. 96 (1989), 221-238.

[5] Busenberg, S., C. Castillo-Chavez, Interaction, pair formation and force of infection terms in sexually-transmitted diseases. In: Mathematical and Statistical Approaches to AIDS Epidemiology (ed. by C. Castillo-Chavez), 289-300. Springer-Verlag, BerlinHeidelberg-New York-London-Paris-Tokyo-Hong Kong 1989.

[6] Busenberg, S., C. Castillo-Chavez, A general solution of the problem of mixing subpopulations, and its application to risk- and age-structured epidemics models for the spread of AIDS. IMA J. Math. Appl. Med. Biol. 8 (1991), 1-29.

[7] Castillo-Chavez, C., S. Busenberg, On the solution of the two-sex mixing problem. In: Proceedings of the International Conference on Differential Equations and Applications to Biology and Population Dynamics (ed. by S. Busenberg, M. Martelli), 80-98. Springer-Verlag, Berlin-Heidelberg-New York-London-Paris-TokyoHong Kong-Barcelona-Budapest 1991.

[8] Castillo-Chavez, C., K.L. Cook, W. Huang, S. A. Levin, On the role of long incubation periods in the dynamics of HIV/AIDS, part 2: multiple group models. In: Mathematical and Statistical Approaches to AIDS Epidemiology (ed. by C. Castillo-Chavez), 200-217. Springer-Verlag, Berlin-Heidelberg-New York-LondonParis-Tokyo-Hong Kong 1989.

[9] Castillo-Chavez, C., S.-F. Shyu, G. Rubin, D. Umbach, On the estimation problem of mixing/pair formation matrices with applications to models for sexually-transmitted diseases. In: AIDS Epidemiology: Methodological Issues (ed. by N.P. Jewell, K. Dietz, V. T. Farewell), 384-402. Birkhäuser, Boston-Basel-Berlin 1992.

[10] Crawford, C.M., S.J. Schwager, C. Castillo-Chavez, A methodology for asking sensitive questions among college undergraduates. Biometrics Unit Technical Report BU-1105-M (1990), Cornell University, Ithaca, New York.

[11] Dietz, K., On the transmission dynamics of HIV. Math. Biosci. 90 (1988), 397-414.

[12] Dietz, K., K.P. Hadeler, Epidemiological models for sexually transmitted diseases. J. Math. Biol. 26 (1988), 1-25. 
[13] Fredrickson, A.G., A mathematical theory of age structure in sexual populations: random mating and monogamous marriage models. Math. Biosci. 20 (1971), 117143.

[14] Gupta, S., R.M. Anderson, R.M. May, Networks of sexual contacts: implications for the patten of spread of HIV. AIDS 3 (1989), 1-11.

[15] Hadeler, K.P., Pair formation in age-structured populations. Acta Applicandae Mathematicae 14 (1989), 91-102.

[16] Hethcote, H.W., J.W. Van Ark, Epidemiological models for heterogeneous populations: proportionate mixing, parameter estimation, and immunization programs. Math. Biosci. 84 (1987), 85-111.

[17] Hethcote, H.W., J.A. Yorke, Gonorrhea Transmission Dynamics and Control. Springer-Verlag, Berlin-Heidelberg-New York-London-Paris-Tokyo-Hong Kong 1984.

[18] Huang, W., K. Cooke, C. Castillo-Chavez, Stability and bifucation for a multiplegroup model for the dynamics of HIV/AIDS transmission. SIAM J. Appl. Math. 52 (1992), 835-854.

[19] Hyman, J.M., E.A. Stanley, The effect of social mixing patterns on the spread of AIDS. In: Mathematical Approaches to Problems in Resource Management and Epidemiology (ed. by C. Castillo-Chavez, S.A. Levin, C.A. Shoemaker), 190-219. Springer-Verlag, Berlin-Heidelberg-New York-London-Paris-Tokyo-Hong Kong 1989.

[20] Jacquez, J.A., C.P. Simon, J. Koopman, L. Sattenspiel, T. Perry, Modeling and analyzing HIV transmission: the effect of contact patterns. Math. Biosci. 92 (1988), 119-199.

[21] Jacquez, J.A., C.P. Simon, J. Koopman, Structured mixing: heterogeneous mixing by the definition of mixing groups. In: Mathematical and Statistical Approaches to AIDS Epidemiology (ed. by C. Castillo-Chavez), 301-315. Springer-Verlag, BerlinHeidelberg-New York-London-Paris-Tokyo-Hong Kong 1989.

[22] Nold, A., Heterogeneity in disease-transmission modeling. Math. Biosci. 52 (1980), 227-240.

[23] Rubin, G., D. Umbach, S.-F. Shyu, C. Castillo-Chavez, Application of capturerecapture methodology to estimation of size of population at risk of AIDS and/or other sexually-transmitted dieseases. Biometrics Unit Technical Report BU-1112-M (1991), Cornell University, Ithaca, New York.

[24] Sattenspiel, L., C. Castillo-Chavez, Environmental context, social interactions, and the spread of HIV. Amer. J. Hum. Biol. 2 (1990), 397-417.

[25] Seber, G.A.F., The Estimation of Animal Abundance and Related Parameters. Macmillan, New York 1982. 